MATEC Web of Conferences 12, 04009 (2014)

DOI: $10.1051 /$ matecconf/20141204009

(C) Owned by the authors, published by EDP Sciences, 2014

\title{
Fatigue-damage localization in steel catenary risers
}

\author{
Víctor F. Hernández ${ }^{1}$, Ramsés Rodríguez ${ }^{2, a}$, Faustino Pérez ${ }^{1}$ and Rommel Burbano ${ }^{1}$ \\ ${ }^{1}$ Instituto Mexicano del Petróleo, Av. 100 m, Mexico \\ ${ }^{2}$ ESIA Zacatenco, Instituto Politécnico Nacional, Av. Juan de Dios Batiz s/n, Mexico
}

\begin{abstract}
Risers used to transport crude oil require Structural Integrity Management plans and programs to allow proper functioning during its design life. Cyclic loading may cause fatigue damage during operation of the riser. Structural Health Monitoring (SHM) is usually applied to detect damages ahead, and be confirmed by non- destructive inspection using remotely operated vehicles. With the information obtained the riser is assessed and if required mitigating measures can be implemented to prevent failure and disasters such as environmental pollution, and human and economic losses. This paper presents a study to locate fatigue damages using signals of the dynamic response. Numerical study cases were defined for a Steel Catenary Riser (SCR) installed in $2000 \mathrm{~m}$ water depth. A damage case was considered, decreasing the stiffness value at a specific location of the structure. Dynamic analyses were performed using a commercial software that incorporates nonlinear behavior. The Modal Slope Difference, Modal Slope Difference and Damage Index methods (with two variations) were applied to locate damage. Based on results the MSID yielded the smallest error values in damage location followed by the Damage Index Method for severity values greater than $6 \%$. Results demonstrate the effectiveness of the proposed methodology to locate fatigue damages in deep-water SCRs.
\end{abstract}

\section{Damage detection methods}

In this paper four damage detection methods were applied in order to locate damage in the structure: the Modal Shape Difference (MShD) and Modal Slope Difference (MSID), Damage Index method (DIM) (Kim and Stubbs, 1993) using mode shapes and first derivative of mode shapes. The first computes the difference between an undamaged and damaged mode shape of the riser, the second a difference between the first derivative of an undamaged and damaged mode shape.

\section{Examples}

In this example the model of a steel catenary riser (SCR) which is located in a water depth of $2000 \mathrm{~m}$ and $2706 \mathrm{~m}$ has curvilinear length was performed. Three damage cases were simulated reducing stiffness values at three specific locations. Dynamic responses of the undamaged and damaged riser were obtained using FLEXCOM and then modal parameters were computed applying the Frequency Domain

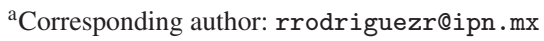

This is an Open Access article distributed under the terms of the Creative Commons Attribution License 4.0, which permits unrestricted use, distribution, and reproduction in any medium, provided the original work is properly cited. 


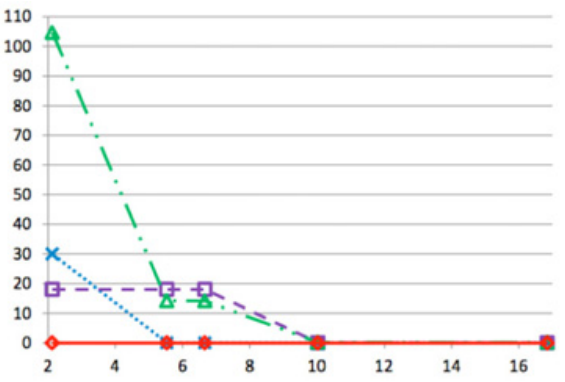

a) Damage at $360 \mathrm{~m}$.

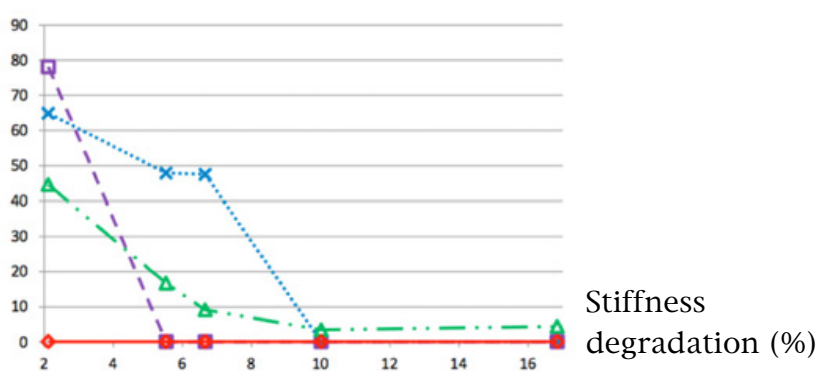

b) Damage at $1344 \mathrm{~m}$.

Figure 1. Relative error of damage location.

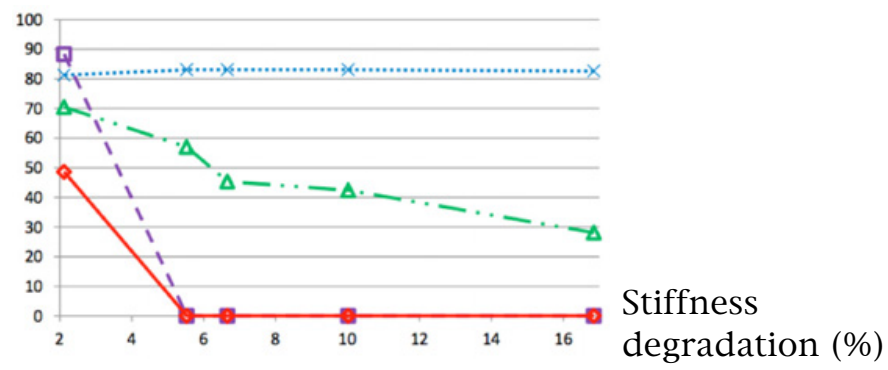

Figure 2. Relative error of damage location, Damage at $2525 \mathrm{~m}$.

Decomposition Method (Brincker et al., 2000). Then the four damage identification methods were applied. Figures 1 and 2 show error values in damage location against stiffness reduction percentages. The blue line corresponds to the DIM, purple for the DIM using first derivative of mode shapes, green for the MShD and red line for the MSID. It can be observed that the MS1D yielded best results for damage location for all three damage cases.

\section{Conclusions}

In this paper four different damage detection methods were applied to a Steel Catenary Riser in deep waters using numerical dynamic response. For all three damage cases the MSID performed best yielding the smallest error values in damage location. Then the Damage Index was also adequate for severity damage values greater than $6 \%$.

The authors would like to acknowledge gratefully Instituto Politécnico Nacional for supporting this research project (20110376) and to Instituto Mexicano del Petróleo for his support with the software. 


\section{FDMDII - JIP 2014}

\section{References}

[1] R. Brincker, L. Zhang, P. Andersen. Modal identification from ambient responses using frequency domain decomposition, IMAC XVIII, San Antonio, Texas (2000)

[2] J.T. Kim, and N. Stubbs. Assessment of the Relative Impact of Model Uncertainty on the Accuracy of Global Nondestructive Damage Detection in Structures. Report prepared for New Mexico State University (1993)

[3] Marine Computation Services Ltd., 2008, FLEXCOM, Galway Technology Park, Galway, Ireland 